\title{
ON QUASINORMAL SUBGROUPS OF CERTAIN FINITELY GENERATED GROUPS
}

\author{
by JOHN C. LENNOX
}

(Received 20th March 1981)

A subgroup $Q$ of a group $G$ is called quasinormal in $G$ if $Q$ permutes with every subgroup of $G$. Of course a quasinormal subgroup $Q$ of a group $G$ may be very far from normal. In fact, examples of Iwasawa show (for a convenient reference see [8]) that we may have $Q$ core-free and the normal closure $Q^{G}$ of $Q$ in $G$ equal to $G$ so that $Q$ is not even subnormal in $G$. We note also that the core of $Q$ in $G, Q_{G}$, is of infinite index in $Q^{G}$ in this example. If $G$ is finitely generated then any quasinormal subgroup $Q$ is subnormal in $G$ [8] and although $Q$ is not necessarily normal in $G$ we have that $\left|Q^{G}: Q\right|$ is finite and $Q^{G} / Q_{G}$ is a nilpotent group of finite exponent [5].

It is our object here to show that if, in addition, $G$ is soluble by finite then $Q^{G} / Q_{G}$ is in fact finite. Thus we have the following theorem.

Theorem A. Suppose that $G$ is a finitely generated soluble by finite group and that $Q$ is a core-free quasinormal subgroup of $G$. Then the normal closure of $Q$ in $G$ is finite.

This means that a quasinormal subgroup of a finitely generated soluble by finite group $G$ is sandwiched between two normal subgroups of $G$ each of finite index in the other. We leave the obvious questions about finitely generated groups open.

We note that in [1] it is shown that if $G$ is a soluble minimax group (not necessarily finitely generated) and $Q$ is a core-free quasinormal subgroup of $G$ closed in the profinite topology on $G$ then $Q^{G}$ is finite. The closure hypothesis on $Q$ given here cannot be dropped as a consideration of the examples of Iwasawa mentioned above reveals.

In [6] Maier and Schmid prove that a core-free quasinormal subgroup of a finite group is contained in the hypercentre of that group. As a consequence of Theorem A we extend their result to the following theorem.

Theorem B. Suppose that $G$ is a finitely generated soluble by finite group which is residually finite and that $Q$ is a core-free quasinormal subgroup of $G$. Then $Q \leqq \zeta_{n}(G)$ for some positive integer $n$.

Here $\zeta_{n}(G)$ denotes the $n$th term of the upper central series of the group $G$.

Thus, in particular, core-free quasinormal subgroups of polycyclic by finite groups (and, indeed, of finitely generated abelian by polycyclic by finite groups) are hypercentral since such groups are residually finite (see [4] and [7]). 
Again, we leave the obvious question for finitely generated soluble groups open. In another direction Busetto has recently shown in [2] that a torsion locally cyclic corefree quasinormal subgroup is always hypercentral.

Proof of Theorem A. Suppose that $G$ and $Q$ are as in the statement of the theorem.

Suppose first of all that there exists no infinite cyclic subgroup of $G$ which intersects $Q$ trivially.

Thus, if $x \in G$, some power of $x$ lies in $Q$ and hence $G / Q^{G}$ is periodic. Now finitely generated periodic soluble by finite groups are finite and hence $G / Q^{G}$ is finite.

Application of a result of Stonehewer [8, Theorem B] yields $\left|Q^{G}: Q\right|$ is finite since $G$ is finitely generated. Hence $|G: Q|$ is finite. But $Q$ is core-free and hence $G$ is finite.

Hence we may assume that some infinite cyclic subgroup of $G$ intersects $Q$ trivially. Then by a result of Gross [3] we have $Q \triangleleft Q^{G}$ and $Q$ is abelian.

Moreover, by Stonehewer [8] $Q^{G} / Q$ is finite and therefore $\left(Q^{G}\right)^{n} \leqq Q$ for some positive integer $n$. Since $Q$ is core-free it follows that $Q^{G}$ and hence $Q$ has finite exponent.

Let $N$ be the normaliser of $Q$ in $G$. Suppose that $x$ is an element of infinite order not in $N$. Then by [8, Lemma 2.1] we have that $\langle x\rangle \cap Q \neq 1$. Hence $x$ has finite order since $Q$ has finite exponent, a contradiction. Hence $x \in G \backslash N$ implies that $x$ has finite order.

We now need a proposition which we state in rather more generality than is necessary since it may be of some independent interest.

Proposition. Suppose that $G$ is a finitely generated soluble by finite group and that $N$ is a subgroup of $G$ such that each element $x \in G$ has some power in $N$. Then $N$ is of finite index in $G$.

Remark. James Wiegold has pointed out that if $A$ is any perfect group and $G$ is the wreath product $A$ wr $Z$, where $Z$ is an infinite cyclic group, then the only subgroups of $G$ which contain powers of all elements of $G$ are of finite index. This example demonstrates that the property described in the proposition is not a property which characterises finitely generated soluble by finite groups among finitely generated groups.

Proof of the proposition. If $G$ is finite there is nothing to prove so we may assume by induction on the derived length of a soluble subgroup of finite index in $G$ that $|G: N A|$ is finite where $A$ is an abelian normal subgroup of $G$. We may therefore assume that $G=N A$ and since in this case $N \cap A \triangleleft G$ we may take $N \cap A=1$. Since $G$ is finitely generated we have a finite set $a_{1} n_{1}, \ldots, a_{s} n_{s}$ of generators for $G$ and a second induction allows us to assume that $G=B N$ where $B=\langle a\rangle^{N}$ for some $a \in A$.

Let $n \in N$ and consider $g=a n$. Then $g^{r} \in N$ for some $r$ so that

$$
a a^{n^{-1}} \ldots a^{n^{-r+1}} n^{r} \in N
$$

Hence

$$
a a^{n^{-1}} \ldots a^{n^{-r+1}} \in N \cap A=1
$$


It follows that

$$
\left[a, n^{r}\right]=1
$$

so that $n^{r} \in C_{N}(a)$, the centraliser in $N$ of $a$.

By the induction hypothesis we have that $\left|N: C_{N}(a)\right|$ is finite and hence $\left[N^{m}, a\right]=1$ for some $m$. We then obtain

$$
\left[N^{m}, B\right]=1 .
$$

But $B$ is then a module for $N / N^{n}$ which is a finite group since $N$ is finitely generated and soluble by finite. Furthermore $a$ has finite order since $a^{s} \in A \cap N=1$ for some $s$. Hence $B$ is finite, since $B / C_{B}(N)$ is finite and $C_{B}(N)$ is finitely $N$-generated and central and so is finite.

It now follows that $|G: N|$ is finite, as required.

Applying the proposition in the situation of Theorem A we have that $|G: N|$ is finite, where $N=N_{G}(Q)$. Hence $Q$ has at most a finite number of conjugates under $G$, say $Q, Q^{g_{1}}, \ldots, Q^{g_{r}}$. We also have that $\left|Q^{G}: Q^{g_{i}}\right|$ is finite for $i=1,2, \ldots, r$ so that $\left|Q Q^{g_{i}}: Q^{g_{i}}\right|$ is finite. Therefore $\left|Q: Q \cap Q^{g_{i}}\right|$ is finite so that $1=Q_{G}=Q \cap Q^{g_{1}} \cap \ldots \cap Q^{g_{r}}$ is of finite index in $Q$. Therefore $Q$ is finite and so $Q^{G}$ is finite since $\left|Q^{G}: Q\right|$ is finite. This completes the proof of Theorem A.

Deduction of Theorem B from Theorem A. By Theorem A we have $Q^{G}$ finite and since $G$ is residually finite there exists a normal subgroup $N$ of finite index in $G$ with $N \cap Q^{G}=1$.

By Maier-Schmid's result applied to $G / N$ we obtain that the repeated commutator $\left[Q^{G},{ }_{n} G\right]$ is contained in $Q N$ for some $n$.

Hence

$$
\left[Q^{G},{ }_{n} G\right] \leqq(Q N) \cap Q^{G}=Q\left(N \cap Q^{G}\right)=Q .
$$

But $\left[Q^{G},{ }_{n} G\right] \triangleleft G$ and so

$$
\left[Q^{G},{ }_{n} G\right]=1
$$

as $Q$ is core-free. Hence $Q \leqq \zeta_{n}(G)$ as required.

\section{REFERENCES}

1. D. C. Brewster and J. C. Lennox, Subnormality and quasinormality in soluble minimax groups, J. Algebra 48 (1977), 368-381.

2. G. BUSETTO, Lattice-theoretical characterizations of hyperabelian groups and of supersoluble groups, To appear.

3. F. Gross, Subnormal, core-free, quasinormal subgroups are solvable, Bull. London Math. Soc. 7 (1975), 93-95. 
4. A. V. Jategaonkar, Integral group rings of polycyclic groups, J. Pure and Appl. Algebra 4 (1974), 337-343.

5. J. C. Lennox, A note on quasinormal subgroups of finitely generated groups, $J$. London Math. Soc., 24 (1981), 127-128.

6. R. MAIER and P. SCHMID, The embedding of quasinormal subgroups in finite groups, Math. Z. 131 (1973), 269-272.

7. J. E. Roseblade, Applications of the Artin-Rees lemma to group rings, Symp. Mathematica 17 (1976), 471-478.

8. S. E. Stonehewer, Permutable subgroups of infinite groups, Math. Z. 125 (1972), 1-16.

University College

Cardiff, Wales 